\title{
Inversion of Isoclinal Matrices
}

\author{
By Leroy J. Derr
}

\begin{abstract}
Triangular matrices (over the reals), whose elements satisfy $a_{i, j}=a_{i+1, j+1}$, can be mapped algebraically into the rational functions to facilitate determination of inverses and factors of the matrices. It is further shown in this note that these methods can be extended to the nontriangular forms to obtain inverses or demonstrate singular cases. Applications are made to certain matrices of Hessenberg type.
\end{abstract}

1. Introduction. Certain matrices of Toeplitz form [5] will be considered as having arbitrary but finite dimension $n \times n$. The matrices will have real elements and the property that $a_{i, j}=a_{i+1, i+1}$ (isoclinal). The purpose of this note is to extend to the nontriangular case methods which have proved useful in obtaining decompositions and inverses for the lower triangular case. Several examples are given, including the inversion of a certain class of Hessenberg matrices.

2. Mathematical Description. Let us first consider the class $V_{n}$ of lower triangular isoclinal (L.T.I.) $n \times n$ matrices. A matrix $A \in V_{n}$ can be denoted by its first column, $\left[a_{0}, a_{1}, \cdots, a_{n-1}\right]$, which defines it completely. The space $V_{n}$ has been shown in [2] to be a commutative vector algebra where the product $A B$ of two elements is given simply by $\sum_{p=0}^{k} a_{p} b_{k-p}, k=0,1, \cdots, n-1$. Hence, if $A \in V_{n}$ is invertible $\left(a_{0} \neq 0\right)$, then $A^{-1}=B$ is easily computed from the recursive system:

$$
a_{0} b_{0}=1, \quad \sum_{p=0}^{k} a_{p} b_{k-p}=0, \quad k=1,2, \cdots, n-1 .
$$

While the above, Eq. (1), are sufficient for the inversion of a given L.T.I. matrix, the problem can frequently arise to seek 'closed' formulas for inverses of such matrices with arbitrary $n$; for example, the first column of the matrix may consist of the first $n$ elements of a given infinite sequence. Before proceeding to the nontriangular case, the following algebraic mapping theorem is given as an aid in such circumstances. Its use will be later illustrated in the examples.

2.1. THEOREM. Let $R$ be the ring of rational functions $\{P(y) / Q(y), Q(0) \neq 0\}$ and let $A \in V_{n}$ with first column $\left[a_{0}, a_{1}, \cdots, a_{n-1}\right]$. Define $e_{n}: V_{n} \rightarrow R$ by $e_{n}\left[a_{0}, a_{1}, \cdots, a_{n-1}\right]$ $=\sum_{p=0}^{n-1} a_{p} y^{p}$; let $p_{n}: R \rightarrow R /\left\langle y^{n}\right\rangle$ be the unique polynomial representation modulo the principal ideal $\left\langle y^{n}\right\rangle$ of $P(y) / Q(y) \in R$ and let $j_{n}: R /\left\langle y^{n}\right\rangle \rightarrow V_{n}$ be $j_{n}\left(\sum_{p=0}^{n-1} c_{p} y^{p}\right)=$ $\left[c_{0}, c_{1}, \cdots, c_{n-1}\right]$. Then, for nonsingular $A, 1 / e_{n}(A) \in R$ and $j_{n} p_{n}\left\{1 / e_{n}(A)\right\}=A^{-1}$.

Proof. Simply note that $A$ is nonsingular if and only if $a_{0} \neq 0$, so $1 / e_{n}(A) \in R$. The mapping $j_{n} p_{n}$ is a ring homomorphism onto and $j_{n} p_{n} e_{n}$ is the identity, so $j_{n} p_{n}\left\{e_{n}(A) / e_{n}(A)\right\}=A \cdot j_{n} p_{n}\left\{1 / e_{n}(A)\right\}=1$.

Such mappings can also be used to factor matrices as shown in [2].

Received September 13, 1971, revised March 6, 1972.

AMS 1970 subject classifications. Primary 15A09; Secondary 20H20.

Copyright @ 1972, American Mathematical Society 
Now, turning to the nontriangular case, we first observe that such an isoclinal matrix can be extended to an L.T.I. matrix by suitable bordering.

2.2. LeMmA. Let $H$ be an $n \times n$ isoclinal matrix such that the $k$ th supra-diagonal is not zero, $k<n$ (and suppose all succeeding supra-diagonals are zero). Then, $H$ may be bordered above and on the right by $k$ rows and columns, by extension of the isoclinal property to form an $(n+k) \times(n+k)$ nonsingular L.T.I. matrix $T$.

Clearly, $T$ is nonsingular since its diagonal is nonzero. Now, multiply $T$ by a suitable permutation matrix $P$, so that $H$ becomes the principal $n \times n$ submatrix of $P T$. The inverse, $(P T)^{-1}=T^{-1} P^{T}$, may now be calculated and the following standard result on matrix partitioning may be applied.

2.3. Lemma. Let $T^{-1} P^{T}$ be partitioned into submatrices $A_{11}, A_{12}, A_{21}, A_{22}$, where $A_{11}$ is the principal $n \times n$ submatrix and $A_{22}$ is $k \times k$. Then, $H^{-1}$ exists if and only if $A_{22}^{-1}$ exists and in this case $H^{-1}=A_{11}-A_{12} A_{22}^{-1} A_{21}$.

A proof may be found in [3].

3. Application. Example 1. We first illustrate the procedure with the simple band matrix $H$ of arbitrary dimension $n \geqq 2$, given by $a_{i, j}=-1,1$, when $i-j=0,-1$, respectively, and zero otherwise. This has one nonzero supra-diagonal, and its companion L.T.I. matrix for Lemma 2.2 is given by its first column as $T=[1,-1,0 \cdots]$. By Theorem 2.1, $T$ is mapped to $1-y$ and $1 /(1-y)$ maps back to give $T^{-1}=$ $[1,1,1 \cdots]$. The required permutation matrix $P$ merely makes the first row of $T$ last, and $T^{-1} P^{T}$ consists of " 1 " below the main diagonal and in the last column, and zero otherwise. By computation from Lemma 2.3, if the elements of $H^{-1}$ are $c_{i, i}$, then $c_{i, j}=-1$ if $i-j \leqq 0$, and zero otherwise.

Example 2. Many matrices of Hessenberg type [4] become singular beyond a certain dimension $n$. A simple example of this is afforded by considering the L.T.I. matrix $T$ whose first column is given by the negative binomial coefficients for $(1+y)^{-3}$, namely, $(-1)^{p}\left(\begin{array}{c}2+p \\ p\end{array}\right), p=0,1, \cdots$. An isoclinal matrix of Hessenberg type $H$ is obtained by deleting the first row and last column of $T$. By applying Theorem 2.1 to $T$, one obtains the first column of $T^{-1}$ as the positive binomial coefficients $[1,3,3,1$, $0 \cdots$. But if $T$ is $n \times n$, then $A_{22}$ from Lemma 2.3 is the $1 \times 1$ matrix whose element is the $n$th coefficient of this sequence. Hence, $H$ is singular when it exceeds $3 \times 3$ in size. Analogous results may be obtained for matrices with more than one nonzero supra-diagonal.

Example 3. In [1], Chow gives formulas for the inverses of a certain class of isoclinal Hessenberg matrices which we shall also do as a further illustration of the above methods. If $\alpha$ and $\delta$ are real numbers, $\alpha \neq 0$, we construct the L.T.I. matrix $T$ whose elements are $a_{i, j}=0$ if $i-j<0,1$ if $i-j=0, \alpha+\delta$ if $i-j=1$ and $\alpha^{i-i}$ if $i-j>1$. The corresponding Hessenberg matrix $H$ is again obtained from $T$ by deleting the first row and last column.

The rational function image of $T$ is $\sum_{p=0}^{\infty} \alpha^{p} y^{p}+\delta y=1 /(1-\alpha y)+\delta y$, and the reciprocal of this function may be simplified to the form:

$$
(1-\alpha y) /\{(1-a y)(1-b y)\},
$$

where $a=D-\delta / 2, b=-D-\delta / 2$ and $D=(1 / 2)\left(\delta^{2}+4 \alpha \delta\right)^{1 / 2}$. We shall treat the case $a \neq b$; the other case involves analogous techniques. The fraction in (I) may be re-expressed by geometric series and multiplied out to give $1+\sum_{q=1}^{\infty} y^{a}\left\{c_{q}-\alpha c_{q-1}\right\}$, 
where $c_{q}=\sum_{p=0}^{q} a^{p} b^{q-p}=\left(a^{q+1}-b^{q+1}\right) /(a-b), q=0,1, \cdots$. The final reduction gives

$$
\{1 /(a-b)\} \sum_{a=0}^{\infty} y^{q}\left\{a^{a}(a-\alpha)-b^{a}(b-\alpha)\right\} .
$$

Hence, the first column of $T^{-1}$ is given by $\phi_{q}=\left\{a^{a}(a-\alpha)-b^{a}(b-\alpha)\right\} /(a-b)$, $q=0,1, \cdots$. If $T$ is $n \times n$, the $1 \times 1$ matrix $A_{2,2}$ of Lemma 2.3 is the element $\phi_{n-1}$, which is not zero in the case we are treating, and $H^{-1}$ may be constructed. If the elements of $H^{-1}$ are $c_{i, k}$ and $\phi_{q}$ is defined to be zero for $q<0$, the result is $c_{i, k}=$ $\left\{-\phi_{i} \cdot \phi_{n-k-1} / \phi_{n}\right\}+\phi_{i-k-1}$. This result was verified on a rainy afternoon by direct multiplication of $H^{-1}$.

The author wishes to acknowledge the several helpful suggestions of the referee, used in preparing the final manuscript.

Mathematics Department

Louisiana State University in New Orleans

New Orleans, Louisiana 70122

1. T. S. CHOw, "A class of Hessenberg matrices with known eigenvalues and inverses," SIAM Rev., v. 11, 1969, pp. 391-395. MR 40 \#5627.

2. L. J. DeRr, "Triangular matrices with the isoclinal property," Pacific J. Math., v. 37, 1971, pp. 41-44.

3. D. K. Faddeev \& V. N. Faddeeva, Computational Methods of Linear Algebra, Fizmatgiz, Moscow, 1960; English transl., Freeman, San Francisco, Calif., 1963, pp. 161-163. MR 28 \#1742; \#4659.

4. A. S. Householder, The Theory of Matrices in Numerical Analysis, Blaisdell, Waltham, Mass., 1964. MR 30 \#5475.

5. I. I. HiRschman, JR. (Editor), Toeplitz Matrices, Studies in Real and Complex Analysis, Math. Assoc. Amer.; Prentice-Hall, Englewood Cliffs, N. J., 1965, pp. 179-209. MR 32 \#1080. 